\title{
Malignant Mediastinal Germ Cell Tumor Stage Grouping of the Pediatric Study Group
}

National Cancer Institute

\section{Source}

National Cancer Institute. Malignant Mediastinal Germ Cell Tumor Stage Grouping of the Pediatric Study Group. NCI Thesaurus. Code C146848.

A term that refers to the staging of mediastinal malignant germ cell tumors according to the Pediatric Study Group staging criteria. This staging system is not an official UICC TNM classification. (WHO Classification of T umors of the Lung, Pleura, Thymus and Heart, 2015) 